\title{
Towards Using LED Arrays for Relative Alignment of Cube Satellite Clusters
}

\author{
Johannes Herrnsdorf ${ }^{1}$, Alexander D. Griffiths ${ }^{1}$, Christopher Lowe ${ }^{2}$, Malcolm Macdonald ${ }^{2}$, Michael J. Strain ${ }^{1}$, \\ and Martin D. Dawson ${ }^{1}$ \\ ${ }^{1}$ Institute of Photonics, Department of Physics, University of Strathclyde, Glasgow G1 1RD (UK), \\ ${ }^{2}$ Department of Mechanical and Aerospace Engineering, University of Strathclyde, Glasgow G1 1XJ (UK) \\ Email: johannes.herrnsdorf@ strath.ac.uk
}

\begin{abstract}
A structured illumination system based on microscale light-emitting diode arrays provides a low size, mass and power approach to monitor the relative angular alignment of nano-satellites operating in cluster configuration.
\end{abstract}

\section{INTRODUCTION}

A range of future space missions are likely to be in the format of clusters of nano-satellites, the potential of which has recently been demonstrated [1]. The alignment and communications between the satellites in a cluster need to be managed by an appropriate network infrastructure. Optical communications have recently enabled extraordinary space communications demonstrations [2] owing to their low size, mass and power requirements, and are therefore attractive for use in inter-satellite links. However, the laser based systems that enabled high data rate transmission are still too bulky to be employed in nano-satellites. Therefore, light-emitting diode (LED) based systems are an attractive alternative for low data rate devices with an extremely small footprint [3], [4]. By employing highly sensitive single photon avalanche diode (SPAD) detectors, it is possible to use LEDs for communications at 10's kb/s over 10's km ranges, while maintaining a low energy consumption compatible with operation onboard a cube satellite [3].

Here, it is shown that, by projecting the output of microscopic LED arrays, it is possible to not only provide pointto-point data transmission, but also to monitor the relative alignment of the satellites. This is particularly important for nano-satellites that, due to their size and weight contraints, do not have highly accurate inertial reference systems.

\section{EXPERIMENTAL SETUP}

This demonstration uses the same setup and modulation format as described by Griffiths et al. [3], with the main difference being that instead of a single LED, a $16 \times 16$ array of LEDs is operated to transmit spatial pattern sequences as described by Herrnsdorf et al. [5] (specifically sequence A therein). A schematic of the principal components is shown in Fig. 1. The LED array is controlled by a field-programmable gate array (FPGA, Opal Kelly XEM3010) and a lens (Thorlabs C220TME-A) is used to project the array such that each LED element is mapped onto a solid angle $\theta$ in the in the projection. Each LED element transmits a 'fingerprint' signal at $2 \mathrm{~kb} / \mathrm{s}$, and the SPAD receiver (Thorlabs SPCM20A) can determine

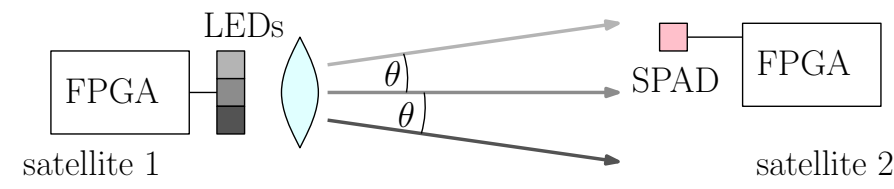

Fig. 1. Schematic of the angular alignment system. Each LED is projected onto a solid angle $\theta$.

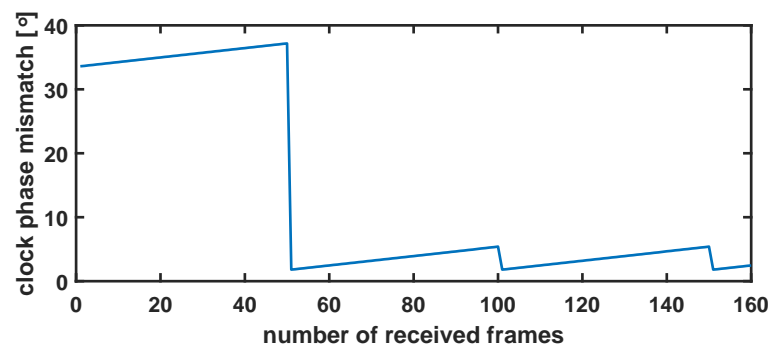

Fig. 2. Phase mismatch of the transmitter and receiver clock for each frame.

the solid angle it is located in by identifying the received modulation 'fingerprint'.

\section{Clock SYNCHRONIZATION}

In order to retrieve the angular alignment position correctly, the read-out electronics of the SPAD receiver need to be synchronized with the LED signal at symbol and frame level. For this purpose, we use a fixed sequence of bits with low selfcorrelation - the clock word. Here, the clock word is 6 bits long and is chosen to be "001101", and is repeated after a 32 bits payload. One frame consists of one clock word followed by the payload.

Fig. 2 shows the effect of symbol level synchronization. This was measured in a Matlab ${ }^{\circledR}$-based implementation, where first a combined frame- and symbol level synchronization was performed, and then symbol level synchronization was updated every 50 frames. The phase difference between the transmitter and receiver clocks could only be measured after the initial frame-level synchronization. Due to the latencies of the data transfer between the Matlab $^{\circledR}$ computation and the FPGA, the mismatch of the two clocks had already accumulated to more than $30^{\circ}$ by the time the first frame is received. However, symbol-level synchronization is still successful, and subsequently the clocks run out of phase by about $5^{\circ}$ before 


\section{a)}

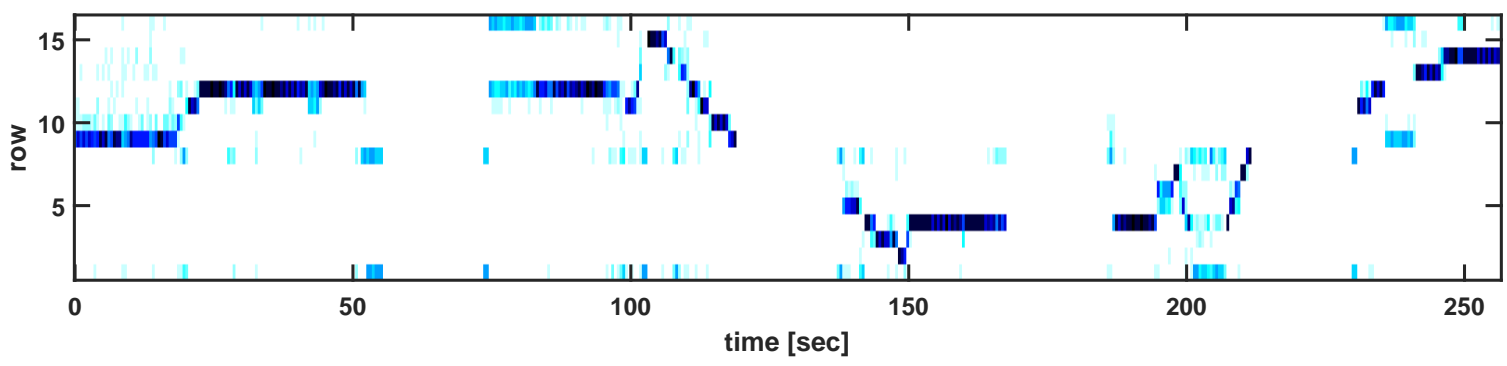

b)

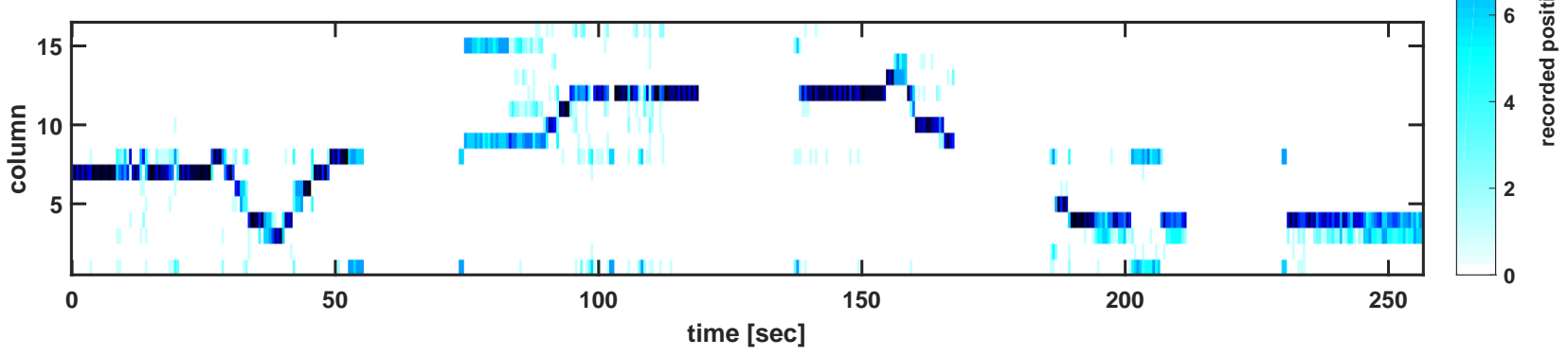

Fig. 3. Pixel coordinates of the SPAD detector recorded by the structured illumination system: $a$ ) row coordinate, and $b$ ) column coordinate. The color coding indicates how many times a coordinate was recorded in a time bin of $0.25 \mathrm{sec}$ duration from 0 times (white) to 14 times (dark blue). Each pixel covers a solid angle of $1.8^{\circ} \times 1.8^{\circ}(31 \mathrm{mrad} \times 31 \mathrm{mrad})$.

being readjusted. This synchronization method has also been implemented on the FPGA thus eliminating the latency issues and, by using 16 frames for synchronization, successful locking was observed at a clock frequency mismatch of $500 \mathrm{ppm}$.

Since we used pulsed encoding of the data [3], the LED driver electronics were observed to be susceptible to ground bounce when many LEDs were active at the same time. This is a limitation of the specific hardware used here and can be eliminated in future versions. As a consequence, the transmission format was modified such that each quadrant of the $16 \times 16$ array had a different frame-level synchronization, and the patterns for alignment detection were distributed over 2 frames.

\section{Alignment Monitoring}

The angular alignment between the LED array and the SPAD detector was deliberately changed while being monitored with the structured light tracking system. A trace of the recorded pixel position, corresponding to the alignment angle between transmitter and receiver, is shown in Fig. 3 . Whenever the alignment moves from one quadrant of the LED array to a neighbouring one, there is a discontinuity of $18 \mathrm{sec}$ duration because the receiver clock has to be synchronized to the clock of the respective quadrant. In the trace shown in Fig. 3, this happens at times $t=56 \mathrm{sec}, 119 \mathrm{sec}, 168 \mathrm{sec}$, and $212 \mathrm{sec}$. Apart from the clock re-synchronization upon moving between quadrants, the trace is continuous and clearly follows the alignment. There are a number of spurious readings. As discussed in [5], the pattern sequence employed here can potentially lead to large jumps in the position reading upon a single bit error in the received signal. For example, from $t=75 \mathrm{sec}$ to $t=86 \mathrm{sec}$, the column reading jumps between columns 9 (true value) and 15 (spurious value). It is known that this issue can be mitigated by using other pattern sequences [5]. Both, the discontinuities of the signal due to clock re-synchronization and the spurious readings are a result of the particular implementation here, using hardware that was not originally designed for this particular purpose, and can therefore be mitigated by dedicated engineering.

In conclusion, the principle of monitoring angular alignment by using structured illumination and single photon sensitive detection has been proven to be effective and readily implemented using FPGA-based digital control. Current efforts focus on the engineering challenges of implementing a flightready system that operates over kilometer distances.

\section{ACKNOWLEDGMENT}

This research was funded by the Engineering and Physical Sciences Research Council under grants $\mathrm{EP} / \mathrm{S} 001751 / 1$ and EP/M01326X/1. Data are available online at https://doi.org/10.15129/8d093a07-27e0-42f0-811f650dd1cbbbd2.

\section{REFERENCES}

[1] C. S. Ruf, C. Chew, T. Lang, M. G. Morris, K. Nave, A. Ridley, and R. Balasubramaniam, Scientific Reports, vol. 8, no. 1, p. 8782, 2018. [Online]. Available: https://doi.org/10.1038/s41598-018-27127-4

[2] B. S. Robinson, D. M. Boroson, D. Burianek, D. Murphy, F. Khatri, A. Biswas, Z. Sodnik, J. Burnside, J. Kansky, and D. M. Cornwell, ser. SpaceOps Conferences, May 2014. [Online]. Available: https://doi.org/10.2514/6.2014-1685

[3] A. Griffiths, J. Herrnsdorf, C. Lowe, M. Macdonald, R. Henderson, M. Strain, and M. Dawson, Materials, vol. 11, no. 9, 92018.

[4] D. N. Amanor, W. W. Edmonson, and F. Afghah, IEEE Transactions on Aerospace and Electronic Systems, vol. 54, no. 6, pp. 2888-2899, Dec 2018.

[5] J. Herrnsdorf, M. Dawson, and M. Strain, IEEE Transactions on Communications, vol. 66, no. 11, pp. 5582 - 5592, 112018. 